\title{
ROSAT-HRI detection of the Class I protostar YLW16A in the $\rho$ Ophiuchi dark cloud
}

\author{
N. Grosso \\ Max-Planck-Institut für extraterrestrische Physik, PO Box 1312, 85741 Garching bei München, Germany
}

Received 17 January 2001 / Accepted 2 March 2001

\begin{abstract}
I analyze unpublished or partially published archival ROSAT data of the $\rho$ Ophiuchi dark cloud. This set of seven overlapping ROSAT HRI pointings, composed of eight one-hour exposures, detects mainly the X-ray brightest $\mathrm{T}$ Tauri stars of this star-forming region. Only two HRI sources are new X-ray sources, and their optical counterparts are proposed as new Weak T Tauri star candidates. Meanwhile the ROSAT HRI caught during just one exposure a weak X-ray source $(\mathcal{L}=10 ; S N R=4.1 \sigma$ for Gaussian statistics $)$ among a group of three embedded young stellar objects including two Class I protostars. Previous ROSAT PSPC, ASCA GIS observations, and as I argue here one Einstein IPC observation, have already detected an X-ray source in this area, but this higher angular resolution data show clearly that X-rays are emitted by the Class I protostar YLW16A. This is the second Class I protostar detected by the ROSAT HRI in this dark cloud. The determination of the intrinsic X-ray luminosity of this event, $L_{\mathrm{X}}[0.1-2.4 \mathrm{keV}]=(9.4-450) 10^{30} \mathrm{erg} \mathrm{s}^{-1}$, critically depends on the source absorption estimate. Improvements will be obtained only by the direct determination of this parameter from fitting of Chandra and XMM-Newton spectra.
\end{abstract}

Key words. open clusters and association: $\rho$ Oph - stars: pre-main sequence - X-rays: stars - infrared: stars

\section{Introduction}

As in the 80's the Einstein X-ray observatory discovered the high X-ray variability of low-mass pre-main sequence stars, called $\mathrm{T}$ Tauri stars, the ROSAT satellite reported in the 90's the first clues of the X-ray activity of younger stellar objects $\left(\sim 10^{5} \mathrm{yr}\right)$, called Class I protostars (Feigelson \& Montmerle 1999). Class I protostars (Lada 1991) are composite objects including a central forming star surrounded by an accretion disk $\sim 10-100 \mathrm{AU}$ in radius, and embedded in an extended infalling remnant envelope of gas and dust up to $\sim 10^{4} \mathrm{AU}$ in size (Shu et al. 1987). Using the Position Sensitive Proportional Counter (PSPC), Casanova et al. (1995) reported in the $\rho$ Ophiuchi star-forming region $(\sim 145 \mathrm{pc}$; de Zeeuw et al. 1999) the detection of seven X-ray sources coinciding with Class I protostars, but possible faint nonprotostellar counterparts were also found in the same error boxes. The ASCA satellite detected more clearly a group of five Class I protostars in the R CrA star-forming region $(\sim 130 \mathrm{pc})$, but the positional uncertainties was quite large $\left(\sim 20^{\prime \prime}\right)$, and the individual source only partially resolved (Koyama et al. 1996). In both cases ROSAT, with its High Resolution Imager (HRI) providing a much better spatial resolution $\left(F W H M \approx 5^{\prime \prime}\right)$, has helped to confirm these

Send offprint requests to: N. Grosso,

e-mail: ngrosso@xray.mpe.mpg.de detections (Grosso et al. 1997; hereafter Paper I; Neuhäuser \& Preibisch 1997). With the goal of preparing the upcoming study of these two star-forming regions with the new generation of X-ray satellites, Chandra and XMM-Newton, their ROSAT observations must be definitively exploited. This work was already done by Neuhäuser \& Preibisch (1997) on R CrA. I study here the unpublished or partially published observations of the $\rho$ Ophiuchi dark cloud to search for X-rays from Class I protostars.

\section{ROSAT observations}

I found in the ROSAT archive that ten overlapping pointings were performed on the $\rho$ Ophiuchi dark cloud: one with the PSPC and nine with the HRI (see Table 1 for the $\log$ of these observations). Among these HRI pointings a sequence of six short exposures ( hour) spreaded over one week, was used by Damiani et al. (1996) to study the X-ray variability of the T Tauri stars SR9 and SR12A-B. One more HRI pointing remains without published results. I thus analyzed these data (8 segments in all; see Table 1) with EXSAS (Zimmermann et al. 1997).

Source detection was performed on each segment separately with the standard command DETECT/SOURCES, which generates a local source detection by a slidingwindow technique followed by a maximum likelihood test, which compares the observed count distribution to a 
Table 1. List of the ROSAT observations of the $\rho$ Ophiuchi dark cloud. Only the segments \#1-8 are analyzed in this letter

\begin{tabular}{|c|c|c|c|c|c|c|c|c|c|}
\hline $\begin{array}{l}\text { ROSAT } \\
\text { archive }\end{array}$ & Proposal name & PI name & $\begin{array}{l}\text { Exp. } \\
{[\mathrm{ks}]}\end{array}$ & $\begin{array}{c}\text { Start } \\
\text { [yymmdd] }\end{array}$ & $\begin{array}{c}\text { End } \\
\text { [yymmdd] }\end{array}$ & $\begin{array}{c}\alpha_{\mathrm{J} 2000} \\
16^{\mathrm{h}}\end{array}$ & $\begin{array}{l}\delta_{\mathrm{J} 2000} \\
-24^{\circ}\end{array}$ & Ref. & $\begin{array}{c}\text { Segment } \\
\#\end{array}$ \\
\hline $200045 p-0$ & $\rho$ Oph Core & Montmerle & 12.9 & 910305 & 913010 & $26^{\mathrm{m}} 31^{\mathrm{s}} .0$ & $31^{\prime} 48^{\prime \prime}$ & (1) & \\
\hline $200045 p-1$ & $\rho$ Oph Core & Montmerle & 19.9 & 910908 & 910908 & $26^{\mathrm{m}} 31^{\mathrm{s}} .0$ & $31^{\prime} 48^{\prime \prime}$ & (1) & \\
\hline $201709 \mathrm{~h}$ & SR9, SR12 & Damiani & 3.3 & 940829 & 940829 & $27^{\mathrm{m}} 28^{\mathrm{s}} .1$ & $31^{\prime} 48^{\prime \prime}$ & $(2)$ & 1 \\
\hline 201710h & SR9, SR12 & Damiani & 5.2 & 940831 & 940831 & $27^{\mathrm{m}} 28^{\mathrm{s}} .1$ & $31^{\prime} 48^{\prime \prime}$ & $(2)$ & 2 \\
\hline 201711h & SR9, SR12 & Damiani & 2.7 & 940901 & 940901 & $27^{\mathrm{m}} 28^{\mathrm{s}} .1$ & $31^{\prime} 48^{\prime \prime}$ & $(2)$ & 3 \\
\hline $201712 \mathrm{~h}$ & SR9, SR12 & Damiani & 5.1 & 940903 & 940903 & $27^{\mathrm{m}} 28^{\mathrm{s}} .1$ & $31^{\prime} 48^{\prime \prime}$ & (2) & 4 \\
\hline 201713h & SR9, SR12 & Damiani & 8.1 & 940904 & 940904 & $27^{\mathrm{m}} 28^{\mathrm{s}} .1$ & $31^{\prime} 48^{\prime \prime}$ & $(2)$ & 5 \\
\hline 201714h & SR9, SR12 & Damiani & 3.7 & 940905 & 940905 & $27^{\mathrm{m}} 28^{\mathrm{s}} .1$ & $31^{\prime} 48^{\prime \prime}$ & $(2)$ & 6 \\
\hline 201618h-1 & ROX20 & Zinnecker & 1.9 & 940917 & 940917 & $27^{\mathrm{m}} 14^{\mathrm{s}} .0$ & $51^{\prime} 36^{\prime \prime}$ & & 7 \\
\hline $201834 \mathrm{~h}$ & Oph Core F & Montmerle & 12.6 & 950309 & 950314 & $27^{\mathrm{m}} 26^{\mathrm{s}} .0$ & $40^{\prime} 48^{\prime \prime}$ & $(3,4)$ & \\
\hline 201618h-2 & ROX20 & Zinnecker & 3.6 & 950817 & 950817 & $27^{\mathrm{m}} 14^{\mathrm{s}} .0$ & $51^{\prime} 36^{\prime \prime}$ & & 8 \\
\hline 201834h-1 & $\rho$ Oph Core F & Montmerle & 27.8 & 950818 & 950820 & $27^{\mathrm{m}} 26.0$ & $40^{\prime} 48^{\prime \prime}$ & $(3,4)$ & \\
\hline $201835 \mathrm{~h}$ & $\rho$ Oph Core A & Montmerle & 51.7 & 950829 & 950912 & $26^{\mathrm{m}} 02^{\mathrm{s}} .0$ & $23^{\prime} 24^{\prime \prime}$ & (4) & \\
\hline 201834h-2 & $\rho$ Oph Core F & Montmerle & 37.5 & 960907 & 960911 & $27^{\mathrm{m}} 26^{\mathrm{s}} .0$ & $40^{\prime} 48^{\prime \prime}$ & (4) & \\
\hline
\end{tabular}

Note: In the ROSAT archive "p" (resp. "h") is for the PSPC (resp. HRI) instrument.

References: (1) Casanova et al. (1995); (2) Damiani et al. (1996); (3) Paper I; (4) Grosso et al. (2000).

model of the point spread function and the local background to discriminate sources from statistical Poissonian background fluctuations. The likelihood of existence, defined as $\mathcal{L}=-\ln P_{0}$ (with $P_{0}$ the probability of the null hypothesis that the observed distribution of counts is only due to a statistical background fluctuation), provides a maximum likelihood measure for the source detection. I accepted only detections with $\mathcal{L} \geq 10(S N R \geq 4.1 \sigma$ for Gaussian statistics) to reduce the number of spurious detections per field to $\sim 0.7-\sim 1.0$. Identification of these $\mathrm{X}$-ray sources was made by cross-correlation with published list of confirmed or suspected cloud members (e.g. André \& Montmerle 1994), IR surveys (e.g. Barsony et al. 1997), and optical catalogue (Monet et al. 1996). To correct X-ray positions for boresight errors, I selected the $\mathrm{X}$-ray sources not associated with protostars having a positional error lower than $2^{\prime \prime}$ both in $\alpha$ and $\delta$, and compared their positions with their IR counterparts in the 2MASS catalogue (second incremental release; Cutri et al. 2000); the mean offsets in $\alpha$ and $\delta$ was then substracted to the $\mathrm{X}$-ray positions of all X-ray sources, and the residual dispersion was quadratically added to the positional errors. Table 2 gives the $\mathrm{X}$-ray source list.

These short exposures detected mainly the X-ray brightest $\mathrm{T}$ Tauri stars of this star-forming region. Only two HRI sources are new X-ray sources. They are located $\sim 25^{\prime}$ south $(\sim 1 \mathrm{pc})$ of the core E/F (Loren et al. 1990) and identified with optical stars ${ }^{1}$ : they are reliable Weak T Tauri star candidates. Meanwhile a weak HRI source $(\mathcal{L}=10)$, detected just in segment 8 , is associated with the IRAS source YLW16 - a group of two Class I protostars (YLW16A; and YLW16B, also called IRS46) and one embedded Classical T Tauri star (GY262). In order to

\footnotetext{
${ }^{1} 11589441(B=14.8, R=12.6) ; 11614157(B=17.9$, $R=14.1)$.
}

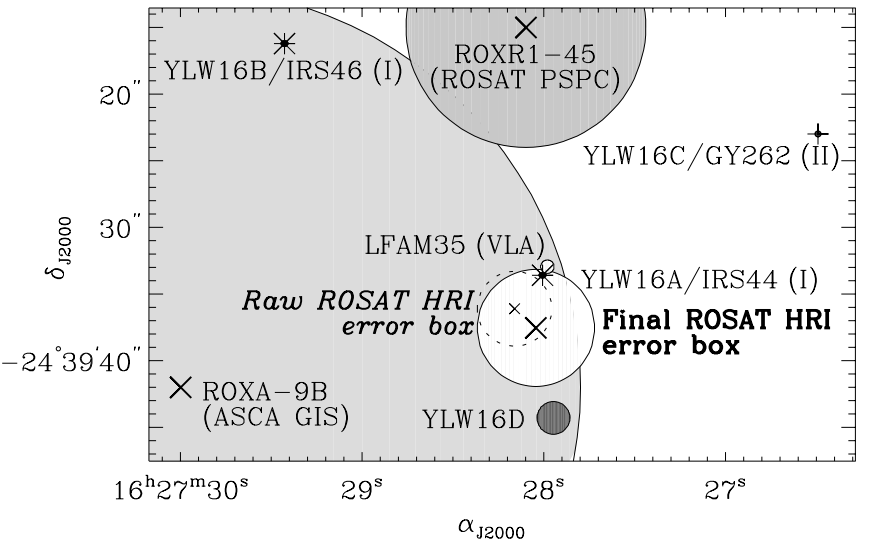

Fig. 1. X-ray detection of the Class I protostar YLW16A with the ROSAT HRI. The positions of the IR sources in this area are from the 2MASS catalogue (Cutri et al. 2000; $\sigma_{2 \mathrm{MASS}} \sim$ $\left.0.3^{\prime \prime}\right)$. The position of YLW16D, given in the Barsony et al. (1997) coordinate frame by Lucas \& Roche (1998), was converted to the 2MASS coordinate frame applying the position offset found for YLW16A in 2MASS compared to Barsony et al. (1997). The position of LFAM35, the VLA detection of YLW16A (Leous et al. 1991), is also shown. The circle radii indicate with the one $\sigma$ positional error of the different observations. The ROSAT HRI error circle after boresight error correction includes only YLW16A

discuss the possible identification of this weak HRI source with protostars, Fig. 1 visualizes the different members of YLW16 in the 2MASS reference frame, to be consistent with my previous astrometric correction. The 2MASS position of YLW16A is within the $4^{\prime \prime}$-radius error circle of the HRI source. Using a Monte-Carlo simulation, I found that the probability that this identification is only due to chance is $1.6 \%$. This reliable identification strenghtens the significance level of this weak HRI detection. 
Table 2. X-ray sources detected by the ROSAT HRI with a likelihood of existence $\geq 10$ in the segments \#1-8. Column (1) gives the IR/optical counterpart name with : GY $=$ Greene \& Young (1989); IRS = Wilking et al. (1989); ROXs = Bouvier \& Appenzeller (1992); SR = Struve \& Rudkjøbing (1949); YLW = Young et al. (1986); numbers are from the PMM USNO-A1.0 catalogue (Monet et al. 1996; the designation prefix 0600-1 was here cut away). Column (2) indicates the IR classification. The better X-ray positions of this segment set in Cols. (3-5) are corrected from boresight errors. The segment number as defined in Table 1 is given in Col. (6). $\mathcal{L}$, in Col. (7), is the likelihood of existence of the X-ray sources for each observation. The count rates are given in the ROSAT $0.1-2.4 \mathrm{keV}$ energy band in Col. (8)

\begin{tabular}{|c|c|c|c|c|c|c|c|}
\hline $\begin{array}{l}\text { Source } \\
\text { Name } \\
(1)\end{array}$ & $\begin{array}{l}\text { IR } \\
\text { Cl. } \\
(2)\end{array}$ & $\begin{array}{c}\alpha_{\mathrm{J} 2000} \\
16^{\mathrm{h}} \\
(3)\end{array}$ & $\begin{array}{c}\delta_{\mathrm{J} 2000} \\
(4)\end{array}$ & $\begin{array}{l} \pm \\
\pm= \\
{\left[{ }^{\prime \prime}\right]} \\
(5)(\end{array}$ & & $\begin{array}{l}\mathcal{L} \\
(7)\end{array}$ & $\begin{array}{c}\text { count rate } \\
{\left[\operatorname{cts~ks}^{-1}\right]} \\
(8)\end{array}$ \\
\hline 1589441 & III? & $? 26^{\mathrm{m}} 04^{\mathrm{s}} .5$ & $-24^{\circ} 57^{\prime} 50^{\prime \prime}$ & 5 & 8 & 54.1 & $20.2 \pm 3.0$ \\
\hline SR24N & II & $26^{\mathrm{m}} 58^{\mathrm{s}} \cdot 3$ & $-24^{\circ} 45^{\prime} 32^{\prime \prime}$ & 3 & $\begin{array}{l}7 \\
8\end{array}$ & $\begin{array}{l}10.8 \\
13.3\end{array}$ & $\begin{array}{l}4.6 \pm 1.7 \\
2.4 \pm 0.9\end{array}$ \\
\hline GY194 & III & $27^{\mathrm{m}} 04^{\mathrm{s}} .8$ & $-24^{\circ} 42^{\prime} 18^{\prime \prime}$ & 5 & 7 & 10.1 & $3.9 \pm 1.6$ \\
\hline ROXs20B & BIII & $27^{\mathrm{m}} 15^{\mathrm{s}} 1$ & $-24^{\circ} 51^{\prime} 40^{\prime \prime}$ & 3 & $\begin{array}{l}7 \\
8\end{array}$ & $\begin{array}{l}18.5 \\
38.0\end{array}$ & $\begin{array}{l}4.0 \pm 1.5 \\
4.5 \pm 1.2\end{array}$ \\
\hline SR12A-B & III & $27^{\mathrm{m}} 19.7$ & $-24^{\circ} 41^{\prime} 39^{\prime \prime}$ & 2 & $\begin{array}{l}1 \\
2 \\
3 \\
4 \\
52 \\
6 \\
7 \\
8\end{array}$ & $\begin{array}{r}143.2 \\
123.0 \\
138.6 \\
128.2 \\
150.2 \\
90.5 \\
42.9 \\
162.3\end{array}$ & $\begin{array}{l}16.7 \pm 2.4 \\
11.3 \pm 1.6 \\
24.2 \pm 3.2 \\
12.3 \pm 1.7 \\
85.4 \pm 3.4 \\
13.0 \pm 2.0 \\
11.7 \pm 2.7 \\
19.0 \pm 2.4\end{array}$ \\
\hline YLW16A & & $27^{\mathrm{m}} 28^{\mathrm{s}} .0$ & $-24^{\circ} 39^{\prime} 38^{\prime \prime}$ & 4 & 6 & 10.0 & $2.2 \pm 0.9$ \\
\hline 1614157 & III? & $27^{\mathrm{m}} 32.5$ & $-25^{\circ} 06^{\prime} 16^{\prime \prime}$ & 6 & $\begin{array}{l}7 \\
8\end{array}$ & $\begin{array}{l}21.3 \\
20.8\end{array}$ & $\begin{array}{r}12.1 \pm 3.1 \\
8.2 \pm 1.9\end{array}$ \\
\hline GY292 & II & $27^{\mathrm{m}} 33^{\mathrm{s}} .1$ & $-24^{\circ} 41^{\prime} 20^{\prime \prime}$ & 5 & $\begin{array}{l}2 \\
4\end{array}$ & $\begin{array}{l}11.8 \\
10.7\end{array}$ & $\begin{array}{l}2.3 \pm 0.8 \\
2.0 \pm 0.7\end{array}$ \\
\hline IRS49 & II & $27^{\mathrm{m}} 38^{\mathrm{s}} .2$ & $-24^{\circ} 36^{\prime} 58^{\prime \prime}$ & 3 & 6 & 10.3 & $31.8 \pm 0.8$ \\
\hline SR9 & II & $27^{\mathrm{m}} 40^{\mathrm{s}} .4$ & $-24^{\circ} 22^{\prime} 01^{\prime \prime}$ & 3 & $\begin{array}{l}1 \\
2 \\
3 \\
4 \\
51 \\
6\end{array}$ & $\begin{array}{l}244.9 \\
582.6 \\
237.5 \\
572.2 \\
012.7 \\
367.0\end{array}$ & $\begin{array}{l}29.5 \pm 3.2 \\
38.7 \pm 2.9 \\
36.7 \pm 3.9 \\
38.4 \pm 2.9 \\
47.5 \pm 2.6 \\
34.8 \pm 3.2\end{array}$ \\
\hline ROXs31 & III & $27^{\mathrm{m}} 52^{\mathrm{s}}$ & $-24^{\circ} 40^{\prime} 53^{\prime \prime}$ & 3 & $\begin{array}{l}1 \\
5 \\
6\end{array}$ & $\begin{array}{l}19.5 \\
29.8 \\
42.1\end{array}$ & $\begin{array}{l}4.8 \pm 1.4 \\
3.6 \pm 0.9 \\
7.4 \pm 1.6\end{array}$ \\
\hline SR20 & III & $28^{\mathrm{m}} 33^{\mathrm{s}} .0$ & $-24^{\circ} 22^{\prime} 55^{\prime \prime}$ & 9 & 1 & 12.0 & $7.2 \pm 2.1$ \\
\hline SR13 & II & $28^{\mathrm{m}} 45^{\mathrm{s}} .5$ & $-24^{\circ} 28^{\prime} 22^{\prime \prime}$ & 5 & $\begin{array}{l}1 \\
2 \\
3 \\
4 \\
5 \\
6\end{array}$ & $\begin{array}{l}33.7 \\
18.9 \\
11.2 \\
96.2 \\
66.4 \\
26.2\end{array}$ & $\begin{array}{l}14.4 \pm 2.7 \\
10.9 \pm 2.2 \\
10.7 \pm 3.0 \\
21.5 \pm 2.5 \\
116.6 \pm 2.0 \\
12.3 \pm 2.5\end{array}$ \\
\hline
\end{tabular}

As shown in Fig. 1, other instruments detected also an X-ray source in this area. The ROSAT PSPC source ROXR1-45 (Casanova et al. 1995) was identified with YLW16. The ASCA GIS source ROXA-9B (Kamata et al. 1997) was only identified with YLW16B, whereas

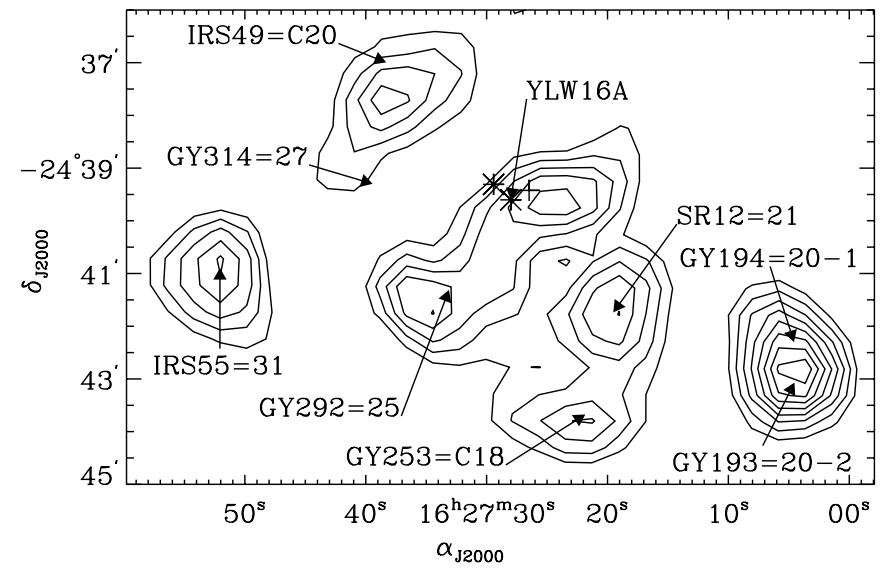

Fig. 2. Einstein IPC detection of the YLW16 IR source group. This intensity contour map is an enlargement of a convolution of the counts distribution (pixel size binned to $30^{\prime \prime}$ ) of an archival Einstein IPC observation of the $\rho$ Ophiuchi dark cloud (8 Sep. 1979; $2.1 \mathrm{ks}$ exposure) with a Gaussian profile $\left(F W H M=1^{\prime}\right)$. The image background is $B=$ $2.510^{-4} \mathrm{cts} \mathrm{s}^{-1} \operatorname{arcmin}^{-2}$. The intensity scale is linear, and was choosen so that the first and fourth contours match the Montmerle et al. (1983)'s contours $n=1$ and 4 , and thus represent levels $2 \times 1.5^{1+n} B$ above standard background. The Einstein IPC $F W H M$ in this area, located $\sim 20^{\prime}$ away from the axis, is larger than $1^{\prime}$. The arrow heads point the 2MASS positions of the IR counterpart of Einstein IPC sources (the ROX number is given after the sign "="). Asterisks (resp. cross) show the Class I protostar (resp. T Tauri star) positions (see also Fig. 1). An Einstein X-ray source is associated with the YLW16 IR source group

YLW16A is also in the ASCA GIS positional error box as mentioned by Carkner et al. (1998); moreover the new IR source discovered by Lucas \& Roche (1998), YLW16D, is in the same error box. An ASCA follow-up observation, made 3.5 years after, did not detect again ROXA9B (Tsuboi et al. 1997): this X-ray source is variable. I noted also in only one of the Einstein IPC observations of Montmerle et al. (1983; see observation I3749.2 Fig. 1.2) an X-ray emission around YLW16, blended with the Einstein X-ray sources associated with SR12 (ROX21) and GY292 (ROX25), which was not in the Montmerle et al.'s detection list. To solve this point, I took from HEASARC the archived screened photon event list ${ }^{2}$, and revisited it using the package XANADU/XIMAGE (also available at HEASARC). I selected only the 8 Sep. 1979 events and energy bins $3-13$ (corresponding to $0.3-8.2 \mathrm{keV}$ ). From this event list, I studied the X-ray source standing near YLW16 with the interactive command sosta, which allows to estimate the background from a nearby area free of sources, and to tune the source box size to exclude events from neighbouring sources. I found for this source a signal to noise ratio $\sim 3.5 \sigma$, and a corrected intensity $S \sim 0.02 \mathrm{cts} \mathrm{s}^{-1}$. Figure 2 shows a contour map constructed from these data. I conclude that the Einstein IPC also detected X-rays from YLW16 (which was not

\footnotetext{
${ }^{2}$ Sum of I3749.1 (8 March 1979), and I3749.2 (8 Sep. 1979).
} 
yet discovered from IR at this time), but the Einstein IPC FWHM $\left(\sim 1^{\prime}\right)$ is too large to find unambiguously the IR counterpart. By contrast to these previous detections with other X-ray instruments, we can see clearly in Fig. 1, thanks to the better angular resolution of the HRI, that the X-ray emission detected by the ROSAT HRI comes only from the Class I protostar YLW16A.

\section{X-ray luminosity of YLW16A}

YLW16A is the second Class I protostar detected by the HRI in the $\rho$ Ophiuchi dark cloud after YLW15 (Paper I). It is remarkable to detect it with only an one-hour HRI exposure, whereas no detection was obtained with a 20 times longer HRI exposure (see Table 1; Grosso et al. 2000). The fact that YLW16A was not detected by the HRI only one day before, with an exposure 2 times longer, implies a variation of its X-ray luminosity by at least a factor 2 in less than one day. This suggests that YLW16A was detected during a state of higher X-ray luminosity, probably due to an X-ray flare, usual in young stellar objects (see Feigelson \& Montmerle 1999). However the source X-ray variability cannot be tested (e.g. with the Kolmogorov-Smirnov test) with only $\sim 8$ cts detected during this short exposure. It is impossible to deduce from this HRI detection whether the previous X-ray observations by other instruments actually detected YLW16A, as this X-ray source is not constant.

The determination of the intrinsic X-ray luminosities of such embedded young stellar object critically depends on the absorption of the X-ray photons by the gas along the line of sight, $N_{\mathrm{H}}$, combination of both the circumstellar material and the interstellar medium. As the HRI has no spectral resolution, no direct information on $N_{\mathrm{H}}$ can be provided by the X-ray event. I thus derive $N_{\mathrm{H}}$ from the source visual extinction due to the dust, $A_{\mathrm{V}}$, assuming the conversion factor $N_{\mathrm{H}}=$ $2.2310^{21} A_{\mathrm{V}} \mathrm{mag} \mathrm{cm}^{-2}$ (Ryter 1996). Applying the methods used in Paper I, I find from near-IR: $A_{\mathrm{V}}=30-40$, and thus $N_{\mathrm{H}}=(6.7-8.9) 10^{22} \mathrm{~cm}^{-2}$. For comparison if I assume that ASCA observed the same source, I have directly from the ASCA spectrum (the only one with enough statistics): $N_{\mathrm{H}}=2.810^{22} \mathrm{~cm}^{-2}$, corresponding to $A_{\mathrm{V}}=13$. Such extinction discrepancies between nearIR and X-ray estimates are not unusual for Class I protostars, but are not well understood (see Kamata et al. 1997), and thus this low value of $N_{\mathrm{H}}$ cannot be excluded. Taking the range $N_{\mathrm{H}}=(2.8-8.9) 10^{22} \mathrm{~cm}^{-2}$ for the absorption, a distance of $145 \mathrm{pc}$, an isothermal RaymondSmith plasma spectrum with a typical protostar flare temperature $k T=4 \mathrm{keV}$, standard solar elemental abundances, I find using W3PIMMS (see HEASARC homepage): $L_{\mathrm{X}}[0.1-2.4 \mathrm{keV}]=(9.4-450) 10^{30} \mathrm{erg} \mathrm{s}^{-1}$ (for comparison $L_{\mathrm{bol}} \sim 13 L_{\odot}$; Wilking et al. 1989), i.e. a factor $\sim 50$ of uncertainties remains in the X-ray luminosity of this event. Nevertheless, this X-ray luminosity is comparable to the one observed during the X-ray triple flare detected from YLW15 by ASCA (Tsuboi et al. 2000).

Improvements of such studies will come from the Chandra and XMM-Newton observations, which will give unambiguous X-ray spectra of YLW16A, and thus will provide an accurate value of $N_{\mathrm{H}}$ to constrain the X-ray luminosity observed by the HRI.

Acknowledgements. I thank the anonymous referee for valuable comments, and T. Montmerle for discussions about his pioneering work with the Einstein observatory. I would like also to thank my host institution, and particularly Prof. J. Trümper and R. Neuhäuser, who have welcomed me for my Marie Curie Individual fellowship supported by the European Union (HPMF-CT-1999-00228).

\section{References}

André, P., \& Montmerle, T. 1994, ApJ, 420, 837

Barsony, M., Kenyon, S. J., Lada, E. A., \& Teuben, P. J. 1997, ApJS, 112, 109

Bouvier, J., \& Appenzeller, I. 1992, A\&AS, 92, 481

Carkner, L., Kozak, J. A., \& Feigelson, E. D. 1998, AJ, 116, 1933

Casanova, S., Montmerle, T., Feigelson, E. D., \& André, P. 1995, ApJ, 439, 752

Cutri, R., Skrutskie, M. F., Van Dyk, S., et al. 2000, http://www.ipac.caltech.edu/2mass/releases/second/ doc/explsup.html

Damiani, F., Micela, G., \& Sciortino, S. 1996, in Röntgenstrahlung from the Universe, ed. H. U. Zimmermann, J. Trümper, \& H. Yorke, MPE Report 263, 27

de Zeeuw, P. T., Hoogerwerf, R., De Bruijne, J. H. J., Brown, A. G. A., \& Blaauw, A. 1999, AJ, 117, 354

Feigelson, E. D., \& Montmerle, T. 1999, ARA\&A, 38, 363

Greene, T. P., \& Young, E. T. 1992, ApJ, 395, 516 (GY)

Grosso, N., Montmerle, T., Feigelson, E. D., et al. 1997, Nature, 387, 56, Paper I

Grosso, N., Montmerle, T., Bontemps, S., André, P., \& Feigelson, E. D. 2000, A\&A, 359, 113

Kamata, Y., Koyama, K., Tsuboi, Y., \& Yamauchi, S. 1997, PASJ, 49, 461

Koyama, K., Hamaguchi, K., Ueno, S., Kobayashi, N., \& Feigelson, E. D. 1996, PASJ, 48, L87

Lada, C. J. 1991, in The Physics of Star Formation and Early Stellar Evolution, NATO ASI, ed. C. J. Lada, \& N. D. Kylafis (Kluwer), 329

Leous, J. A., Feigelson, E. D., André, P., \& Montmerle, T. 1991, ApJ, 379, 683

Loren, R. B., Wootten, A., \& Wilking, B. A. 1990, ApJ, 365, 269

Lucas, P. W., \& Roche, P. F. 1998, MNRAS, 299, 699

Monet, D., et al. 1996, USNO-SA1.0 catalogue, U.S. Naval Observatory, Washington DC

Montmerle, T., Koch-Miramond, L., Falgarone, E., \& Grindlay, J. E. 1983, ApJ, 269, 182

Neuhäuser, R., \& Preibisch, T. 1997, A\&A, 322, L37

Ryter, C. 1996, Ap\&SS, 236, 285

Shu, F. H., Adams, F. C., \& Lizano, S. 1987, ARA\&A, 25, 23

Struve, O., \& Rudkjöbing, M. 1949, ApJ, 109, 92

Tsuboi, Y., Imanishi, K., Koyama, K., Grosso, N., \& Montmerle, T. 2000, ApJ, 532, 1089

Wilking, B. A., Lada, C. J., \& Young, E. T. 1989, ApJ, 340, 823

Young, E. T., Lada, C. J., \& Wilking, B. A. 1986, ApJ, 340, 823

Zimmermann, H. U., Böse, G., Becker, W., et al. 1997, EXSAS User's Guide, ROSAT SDC, Garching 